growth (a class of functions discussed in Chapter 5). This will undoubtedly continue.

The book by Klimek gives an excellent introduction to the subject of maximal plurisubharmonic functions. He develops the theory in detail from scratch, making the book suitable for use in a graduate course. The first two chapters have exercises at the end. It would have been useful to also have exercises after the remaining chapters.

J. E. ForNAESS

UNIVERSITY OF MICHIGAN

E-mail address: fornaess@math.lsa.umich.edu

BULLETIN (New Series) OF THE

AMERICAN MATHEMATICAL SOCIETY

Volume 28, Number 2, April 1993

(c) 1993 American Mathematical Society

$0273-0979 / 93 \$ 1.00+\$ .25$ per page

Analysis of Toeplitz operators, by A. Böttcher and B. Silbermann. SpringerVerlag, New York, 1990, 512 pp., \$79.00. ISBN 3-540-52147-X

Given a complex-valued function $\phi$ on the real line $\mathbf{R}$, one can define the multiplication operator $M(\phi)$, acting on the Lebesgue space $L^{2}(\mathbf{R})$, by the recipe $M(\phi): f \rightarrow \phi f$. To operator theorists these objects are useful and well understood, yet not without a certain charm due to a nice correspondence between properties of $M(\phi)$ and properties of $\phi$. If you are a Fourier analyst, you will probably want to multiply "in the Fourier transform variable" as well, via the Fourier multiplier $D(\psi)=F^{-1} M(\psi) F$, where $F$ is the Fourier transform on $L^{2}(\mathbf{R})$. Since $F$ is unitary (thus preserving all Hilbert space structure), $D(\psi)$ and $M(\psi)$ are isomorphic as Hilbert space operators, but they relate very differently to the functional values of vectors in $L^{2}(\mathbf{R})$. On taking algebraic combinations of $M(\phi)$ 's and $D(\psi)$ 's, one has an interesting mix indeed, including the linear differential operators with variable coefficients: if $\psi(x)=x$, then $D(\psi)=-i d / d x$. If you want to study bounded operators on $L^{2}(\mathbf{R})$, you must take $\phi$ and $\psi$ in $L^{\infty}(\mathbf{R})$, but you are still left with bounded pseudodifferential operators, including several famous subclasses (all of which are generated by restricting either $\phi$ or $\psi$ to be $\chi$, the characteristic function of $[0, \infty))$ : singular integral operators, Wiener-Hopf operators, Hankel operators, and, the subject of the book of Böttcher and Silbermann, the Toeplitz operators

$$
D(\chi) M(\phi) D(\chi) .
$$

For most purposes, the preferred habitat of Toeplitz operators is the unit circle $\mathbf{T}$, where they look a bit more natural. Let us write $L^{2}$ for the Lebesgue space on $\mathbf{T}$ (with respect to normalized arc-length measure $d \theta / 2 \pi$ ), which comes equipped with the orthonormal basis $\left\{e^{i n \theta}: n=0, \pm 1, \pm 2, \ldots\right\}$. The Hardy subspace $H^{2}$ is the closed linear span of the nonnegative frequencies $\left\{e^{i n \theta}: n=0,1,2, \ldots\right\}$. The orthogonal projection $P$ of $L^{2}$ onto $H^{2}$ is the analogue of $D(\chi)$ above, and the analogue of (1) is $P M(\phi) P$, where $\phi$ is now an $L^{\infty}$ function on $T$. Since the last operator annihilates the orthogonal 
complement of $H^{2}$, it is customary to restrict it to $H^{2}$ and define the Toeplitz operator $T(\phi): H^{2} \rightarrow H^{2}$ by the rule $T(\phi) f=P(\phi f)$. The matrix of $T(\phi)$ with respect to the nonnegative frequencies is

$$
\left(\begin{array}{cccc}
a_{0} & a_{-1} & a_{-2} & \cdots \\
& & & \ddots \\
a_{1} & a_{0} & a_{-1} & \\
& & & \ddots \\
a_{2} & a_{1} & a_{0} & \\
\vdots & \ddots & \ddots & \ddots
\end{array}\right)
$$

where $\left\{a_{n}\right\}$ are the Fourier coefficients of $\phi$. These are the Toeplitz matrices, considered by Otto Toeplitz in the early twentieth century.

Toeplitz operators and their isomorphs, the Wiener-Hopf operators, have a number of applications, for example to stationary stochastic processes and orthogonal polynomials, as well as diffraction theory, transport theory, and other subjects in mathematical physics. They are, however, especially attractive as objects in pure mathematics, and their study has brought about a felicitous convergence of functional analysis and function theory.

\section{EARLY THEOREMS}

The study of $T(\phi)$ in the modern mode dates from the 1950s, coinciding with the first flowering of nonselfadjoint operator theory. The key problem was, and remains, to decide when $T(\phi)$ is invertible, or equivalently since $T(\phi)-\lambda I=T(\phi-\lambda)$, to find the spectrum $\operatorname{sp} T(\phi)$ in terms of $\phi$, the symbol of $T(\phi)$.

Hartman and Wintner [21] showed that in the selfadjoint case (the case when $\phi$ is real valued), one has $\operatorname{sp} T(\phi)=$ [ess inf $\phi$, ess $\sup \phi$ ], in contrast to $M(\phi)$, whose spectrum is merely the essential range of $\phi$. This result is a lovely illustration of (and is implied by) three properties of the spectrum which hold for arbitrary $\phi$. They are as follows: the spectrum always contains the essential range of $\phi$ (Hartman and Wintner [20]), the spectrum is contained in the closed convex hull of the essential range (Brown and Halmos [6] and Devinatz [13]), and the spectrum is always connected (Widom [39]). Douglas has written [16, p. 196] that Widom's proof "gives no hint as to why the result is true"; that no more intuitive argument has been found in almost thirty years underscores the difficulty of the invertibility question.

In fact, Widom [38] and Devinatz [13] independently gave exact invertibility criteria, which have been of considerable theoretical utility but which are hard to check in practice. According to the authors of Analysis of Toeplitz operators (p. 60):

This is the reason for a great part of all further investigations devoted to the invertibility of Toeplitz operators. The main goal of these investigations is to obtain invertibility criteria, or equivalently, descriptions of the spectrum, in terms of geometric data of the symbol. The Widom-Devinatz theorems answer the question in analytical language. 


\section{Algebra}

To confront the "geometric data of the symbol", one can start with some algebra. The map $\phi \rightarrow T(\phi)$, which we can call $T$, carries $L^{\infty}$ into $\mathscr{L}\left(H^{2}\right)$, the $C^{*}$-algebra of bounded operators on $H^{2}$. It is linear, norm-preserving, and a *-map: $T(\bar{\phi})=T(\phi)^{*}$. However, $T$ does not (fortunately) preserve products; without this apparent defect, the theory of $T(\phi)$ would be much less interesting.

The discovery of such multiplicative properties as $T$ possesses has provided one key to the analysis of $T(\phi)$. Brown and Halmos [6] showed that $T(\psi \phi)=$ $T(\psi) T(\phi)$ exactly when either $\phi$ or $\bar{\psi}$ is analytic, that is, belongs to the algebra $H^{\infty}=L^{\infty} \cap H^{2}$. A weaker kind of multiplicativity involves the ideal $\mathscr{K}$ of compact operators in $\mathscr{L}\left(H^{2}\right)$. Let $\pi$ denote the quotient map from $\mathscr{L}\left(H^{2}\right)$ to the Calkin algebra $\mathscr{L}\left(H^{2}\right) / \mathscr{K}$, and write $T^{\pi}$ for the composition $\pi \circ T$. When does $T^{\pi}(\psi \phi)=T^{\pi}(\psi) T^{\pi}(\phi)$, that is, when is $T(\psi \phi)-T(\psi) T(\phi)$ compact? This hard problem was solved by the combined efforts of Axler, Chang, and Sarason [3], and Vol'berg [36]. However, a special case observed much earlier by Coburn [9], and implicit in the work of Gohberg and Krein [18], is especially useful: $T^{\pi}$ is multiplicative on the algebra $C$ of continuous functions.

Recall that an operator $A$ on $H^{2}$ is Fredholm if its image in $\mathscr{L}\left(H^{2}\right) / \mathscr{K}$ is invertible. Equivalently, $A$ has closed range and both $\operatorname{ker} A$ and $\operatorname{ker} A^{*}$ are finite dimensional; the difference of the two dimensions is the Fredholm index, ind $A$. Now if $B$ is a subset of $L^{\infty}$, let us write $\mathscr{T}(B)$ for the norm-closed algebra in $\mathscr{L}\left(H^{2}\right)$ generated by those $T(\phi)$ with $\phi$ in $B$. It is then a consequence of Coburn's result and some elementary $C^{*}$-algebra theory that $\mathscr{T}(C)$ contains $\mathscr{K}$ and that $T^{\pi}: C \rightarrow \mathscr{T}(C) / \mathscr{K}$ is an isometric *-isomorphism. It follows that, for continuous $\phi, T(\phi)$ is Fredholm if and only if $\phi$ never vanishes on the circle. A homotopy argument then shows that ind $T(\phi)$ is the negative of the winding number of $\phi$ about the origin. The final step to invertibility is provided by a lemma of Coburn, valid for any Toeplitz operator, which asserts that either $\operatorname{ker} T(\phi)$ or $\operatorname{ker} T(\phi)^{*}$ is the zero subspace. Thus ind $T(\phi)=0$ is equivalent to invertibility. This theorem, in various versions, is due to Krein, Calderon, Spitzer, Widom, and Devinatz. The elegant argument sketched above was found by Coburn and Douglas; see [16].

It is a short step from the preceding remarks to see that $T^{\pi}$ is actually multiplicative on the vector sum $H^{\infty}+C$. This interesting space, shown by Sarason to be a closed subalgebra of $L^{\infty}$, has come to occupy a central place in the theory. Douglas [16] discovered that the invertibility theorem above holds for $H^{\infty}+C$ provided "nonvanishing" and "winding number" are suitably interpreted.

\section{Discontinuities AND localization}

The natural question is how to proceed with discontinuous $\phi$ lying in algebras (such as $L^{\infty}$ itself) on which $T^{\pi}$ is not multiplicative. One approach is to look at points of discontinuity under a microscope, as it were, via the Gelfand theory. Consider $L^{\infty}$ as a $C^{*}$-algebra. It possesses a space $\mathscr{M}\left(L^{\infty}\right)$ of multiplicative linear functionals, sometimes called the maximal ideal space. The Gelfand transform associates to each $\phi$ in $L^{\infty}$ a continuous function $\hat{\phi}$ on $\mathscr{M}\left(L^{\infty}\right)$ via the formula $\hat{\phi}(\alpha)=\alpha(\phi)$. The map $\phi \rightarrow \hat{\phi}$ is a ${ }^{*}$-isomorphism of $L^{\infty}$ and 
the space of continuous functions on $\mathscr{M}\left(L^{\infty}\right)$. Now the maximal ideal space $\mathscr{M}(C)$ of $C$ consists of just the evaluation functionals at points $\lambda$ in $\mathbf{T}$. One defines the fiber $\mathscr{M}_{\lambda}\left(L^{\infty}\right)$ over $\lambda$ to be the set of those $\alpha$ in $\mathscr{M}\left(L^{\infty}\right)$ which agree on $C$ with evaluation at $\lambda$. This set can be thought of as an exploded version of the point $\lambda$ with the restriction $\hat{\phi} \mid M_{\lambda}\left(L^{\infty}\right)$ encoding the nature of the discontinuity of $\phi$ at $\lambda$. In particular, the essential cluster set of $\phi$ at $\lambda$ equals the range of $\hat{\phi}$ on $\mathscr{M}_{\lambda}\left(L^{\infty}\right)$.

Now suppose that $B$ is a $C^{*}$-subalgebra of $L^{\infty}$ containing $C$ and that we want to understand $T(\phi)$ for $\phi$ in $B$. For each $\lambda$ in $T$, let $I_{\lambda}$ denote the closed ideal in $\mathscr{T}(B)$ generated by those $T(\phi)$ with $\phi$ in $C$ and $\phi(\lambda)=0$. The key point, discovered by Douglas [16], is that $T(\phi)$ is Fredholm exactly when each "local operator" $T_{\lambda}(\phi)$, defined to be the abstract coset $T(\phi)+I_{\lambda}$, is invertible in $\mathscr{T}(B) / I_{\lambda}$. If we take $B=L^{\infty}$, some pretty theorems of a very general nature emerge. For example, the local operator $T_{\lambda}(\phi)$ depends only on the restriction $\hat{\phi} \mid M_{\lambda}\left(L^{\infty}\right)$. Hence, $T(\phi)$ is Fredholm precisely when, for each $\lambda$, there exists $\psi$ in $L^{\infty}$ with $T(\psi)$ Fredholm and $\hat{\psi}=\hat{\phi}$ on $M_{\lambda}\left(L^{\infty}\right)$. This is one version of a principle of Simonenko, Douglas, and Sarason: Fredholmness of $T(\phi)$ is entirely a local property of $\phi$, in contrast to invertibility, which depends on a global invariant, the Fredholm index.

If the above seems abstract, small $B$ can yield beautifully concrete results. Suppose $B=P C$, the closed algebra generated by the piecewise continuous functions. Functions in $P C$ have right- and left-hand limits at every point in T. Although $T^{\pi}$ is not multiplicative on $P C, \mathscr{T}(P C) / \mathscr{K}$ is commutative and each local algebra $\mathscr{T}(P C) / I_{\lambda}$ is isomorphic to $C([0,1])$ via the mapping which carries $T_{\lambda}(\phi)$ to the function $t \phi\left(\lambda_{+}\right)+(1-t) \phi\left(\lambda_{-}\right), 0 \leq t \leq 1$, where $\phi\left(\lambda_{+}\right)$and $\phi\left(\lambda_{-}\right)$are the right- and left-hand limits of $\phi$ at $\lambda$. It is immediate that the spectrum of the local operator $T_{\lambda}(\phi)$ is the complex line segment $\left[\phi\left(\lambda_{-}\right), \phi\left(\lambda_{+}\right)\right]$; hence, $T(\phi)$ is Fredholm exactly when no such segment (including the degenerate ones) contains the origin. The winding number of the image of $\phi$ augmented by the nondegenerate segments gives -ind $T(\phi)$. This result, which strikingly illustrates the connectedness of $T(\phi)$, was discovered by Widom [37], Simonenko [33], Devinatz [13], and Gohberg [17]. The local algebras were computed by Douglas [15] and Silbermann [32].

The inner workings of localization require that $\mathscr{T}(C) / \mathscr{K}$ be contained in the center of $\mathscr{T}(B) / \mathscr{K}$. However, algebras larger than $C$ have this property, and localization over them yields smaller fibers and, thus, a finer theory. A beautiful example of this was treated by Sarason [31] with $C$ replaced by the quasi-continuous functions $Q C$, the intersection of $H^{\infty}+C$ with its complex conjugate. Since $H^{\infty}$ intersects its complex conjugate only in the constant functions, it is surprising that $Q C$ is considerably larger than $C$ and, in fact, coincides with the bounded functions of vanishing mean oscillation. It is a result of Douglas that $\mathscr{T}(Q C) / \mathscr{K}$ actually is the center of $\mathscr{T}\left(L^{\infty}\right) / \mathscr{K}$. For $B$, take $P Q C$, the closed algebra in $L^{\infty}$ generated by $P C$ and $Q C$. If $\xi$ is in $\mathscr{M}(Q C)$, the fiber $\mathscr{M}_{\xi}(P Q C)$ (defined like the $L^{\infty}$ fibers above, but with $P Q C$ replacing $L^{\infty}$ and $\xi$ playing the role of the evaluation functional at $\lambda$ ) sometimes contains an element $\xi_{+}$, sometimes another $\xi_{-}$, and sometimes both, but never more than these; it is analogous to the more concrete two-point fiber $\mathscr{M}_{\lambda}(P C)$, for $\lambda$ in $\mathbf{T}$, which consists of the right and left limit functionals 
at $\lambda$. For $\phi$ in $P Q C$, Fredholmness of $T(\phi)$, interpreted locally, means that the segments $\left[\hat{\phi}\left(\xi_{-}\right), \hat{\phi}\left(\xi_{+}\right)\right]$never hit the origin. Remarkably, Sarason was able to describe $\mathscr{M}(P Q C)$ concretely enough so as to cast this in terms of symbol geometry: $T(\phi)$ is Fredholm exactly when the segments

$$
\left[\frac{1}{\delta} \int_{\theta-\delta}^{\theta} f\left(e^{i t}\right) d t, \frac{1}{\delta} \int_{\theta}^{\theta+\delta} f\left(e^{i t}\right) d t\right]
$$

connecting the left- and right-hand averages of $\phi$ at $\lambda=e^{i \theta}$ stay away from zero as $\delta \downarrow 0$, uniformly in $\lambda$.

\section{OPERATORS ON THE LINE}

Local algebras take an interesting turn on the real line. Power [26], motivated by spectral results of Duduchava, showed that Douglas's local theory can be used to describe, in principle, the Fredholm status of any finite algebraic combination of $M(\phi)$ 's and $D(\psi)$ 's, where $\phi$ and $\psi$ lie in $P C$ on the line (such functions also have limits at $-\infty$ and $+\infty)$. Let $\Psi(P C)$ denote the $C^{*}$-algebra generated by all such $M(\phi)$ and $D(\psi)$. A fascinating point is that $\Psi(P C) / \mathscr{K}$, unlike $\mathscr{T}(P C) / \mathscr{K}$ on the circle, is not commutative. Replacing $P C$ by $C$, the algebra of continuous functions on $\mathbf{R}^{*}=\mathbf{R} \cup\{\infty\}$, yields a smaller operator algebra $\Psi(C)$ such that $\Psi(C) / \mathscr{K}$ lies in the center of $\Psi(P C) / \mathscr{K}$ and has maximal ideal space which can be realized as $\mathscr{M}=\left(\mathbf{R}^{*} \times \mathbf{R}^{*}\right) \backslash(\mathbf{R} \times \mathbf{R})$. For $(x, y)$ in $\mathscr{M}$, the local algebra is $\Psi(P C) / I_{(x, y)}$, where $I_{(x, y)}$ is the closed ideal in $\Psi(P C)$ generated by all $M(\phi)$ and $D(\psi)$ with $\phi$ and $\psi$ in $C$ and $\phi(x)=\psi(y)=$ 0 . For points $(x, y)$ other than $(\infty, \infty)$ the corresponding local operators $M_{(x, y)}(\phi)$ and $D_{(x, y)}(\phi)$ do not in general commute, and, in fact, $\Psi(P C) / I_{(x, y)}$ is *-isomorphic to the $C^{*}$-algebra of $2 \times 2$ matrices over $C([0,1])$ generated by the idempotents

$$
p=\left(\begin{array}{ll}
1 & 0 \\
0 & 0
\end{array}\right) \text { and } q=\left(\begin{array}{cc}
t & \sqrt{t(1-t)} \\
\sqrt{t(1-t)} & 1-t
\end{array}\right) .
$$

This can lead to relatively exotic (but computable) local spectra. For general $L^{\infty}$ symbols, however, very little is known.

Simple combinations of $M(\phi)$ 's and $D(\psi)$ 's yield a menagerie of classical operators. If $a(x, y)$ is a finite sum of terms like $\phi(x) \psi(y)$, the corresponding sum $S$ of the operators $M(\phi)(I-2 D(\chi)) M(\psi)$ is the singular integral operator given by

$$
S f(x)=\frac{1}{\pi i} \int_{-\infty}^{\infty} \frac{a(x, y)}{x-y} f(y) d y,
$$

the integral being taken in the principal value sense. The operator $D(\chi)$ itself is exactly the orthogonal projection $P_{+}$onto the Hardy space $H_{+}^{2}$ on $\mathbf{R}$, an analogue of $H^{2}$ on $\mathrm{T}$, and the image of $L^{2}(0, \infty)$ under $F^{-1}$. The WienerHopf operator $W$ on $L^{2}(0, \infty)$, given by

$$
W f(x)=\int_{0}^{\infty} k(x-t) f(t) d t
$$

with $k$ integrable on $\mathbf{R}$, is the restriction of $M(\chi) D(\phi) M(\chi)$ to $L^{2}(0, \infty)$, where $\phi=\sqrt{2 \pi} F^{-1} k$. That Wiener-Hopf operators are isomorphic to Toeplitz 
operators and that Toeplitz operators on $\mathbf{R}$ and $\mathbf{T}$ are the same up to isomorphism was settled by Rosenblum and Devinatz, thus unifying parallel theories. The equivalences are given by the Fourier transform and a change of variables from $\mathbf{R}$ to $\mathbf{T}$. The line, however, seems to be best for studying certain phenomena, particularly oscillatory discontinuities with the discontinuity placed at $\infty$, for example as in Coburn-Douglas [10] and Abrahamse [2]. Finally, the Hankel operators, which in one incarnation take the form $\left(I-P_{+}\right) M(\phi) P_{+}$, should be mentioned. They have a large literature and many applications, as well as close connections (ignored here). to Toeplitz operators; see, for example, Power [27] and Peller [25].

\section{But, WhAT is $T(\phi)$ ?}

The results above belong mainly to Fredholm theory, or behavior modulo the compact operators, with almost accidental implications for invertibility via Coburn's lemma. It is reasonable to go further and ask how $T(\phi)$ moves vectors around in $H^{2}$. Answers are scarce. For selfadjoint operators, the spectral multiplicity theory of Hellinger and Hahn is available to answer this question in principle. Given a selfadjoint $T(\phi)$, there is a direct integral Hilbert space

$$
\mathscr{D}=\int_{\operatorname{sp} T(\phi)} \oplus H_{\lambda} d \nu(\lambda)
$$

(an $L^{2}$-like space of vector-valued functions $f$ whose values $f(\lambda)$ are constrained to lie in the variable Hilbert spaces $H_{\lambda}$ at each $\lambda$ ) and a unitary operator $U: H^{2} \rightarrow \mathscr{D}$ such that $U T(\phi) U^{-1}$ is the simple-looking multiplication operator $f(\lambda) \rightarrow \lambda f(\lambda)$. The multiplicity function $n(\lambda)=\operatorname{dim} H_{\lambda}$ and the measure class of $\nu$ determine $T(\phi)$ up to isomorphism. Rosenblum [29] showed that for Toeplitz operators $\nu$ is always Lebesgue measure on $\operatorname{sp} T(\phi)$, while in [22] Ismagilov announced a formula for $n(\lambda)$. Working independently, Rosenblum [28] discovered the same formula and supplied a proof, including an explicit representation for $U$, thus completing the selfadjoint theory little more than a decade after Hartman and Wintner described the spectrum.

Multiplicity theory is also available for normal operators (those which commute with their adjoints), but this does not take one much further with Toeplitz operators: the only normal $T(\phi)$ are affine functions of selfadjoints. Clark and Morrel, however, posed and answered a fruitful question [7, 8]. Suppose $\phi$ is continuous and real valued, so that it maps $\mathbf{T}$ onto a closed curve which looks like a rubber band squashed flat on the real axis. If one teases the rubber band apart into a curve in the plane, possibly self-intersecting, does Rosenblum's mapping $U: H^{2} \rightarrow \mathscr{D}$ continue to exist in some distorted way? They showed that when $\phi$ is the restriction of a rational function to $\mathbf{T}, \mathscr{D}$ is transformed into a direct sum of copies of $\mathrm{H}^{2}$ and possibly another direct integral, while $U$ persists as an invertible (but no longer unitary) operator. If, for example, $\phi$ sweeps out a figure eight, traversed once except for the double point, then $U T(\phi) U^{-1}=T(a) \oplus T(b)^{*}$ acting on $H^{2} \oplus H^{2}$, where $a$ and $b$ are respectively the boundary functions of conformal maps of the unit disk onto the two regions surrounded by the loops of $\phi$, the first with positive orientation, the second with negative. This is a major step in understanding $T(\phi)$ because analytic Toeplitz operators (those with symbols in $H^{\infty}$ like $T(a)$ and $T(b)$ ) are simpler objects than Toeplitz operators in general; see below. 
A final case links Toeplitz operators, subnormal operators, and weighted shifts in an unexpected way. An operator $S$ on a Hilbert space $H$ is subnormal if there exists a Hilbert space $K$ containing $H$ and a normal operator $N$ on $K$ which leaves $H$ invariant and whose restriction to $H$ is $S$. This class has deep and well-developed connections with function theory [11]. Normal operators are of course subnormal. Among the Toeplitz operators, the analytic ones are also subnormal: any $M(\phi)$ acting on $L^{2}$ is normal, and when $\phi$ is in $H^{\infty}$, $M(\phi)$ leaves $H^{2}$ invariant and so $T(\phi)=M(\phi) \mid H^{2}$. Note that the projection $P$ can be omitted here from the definition of $T(\phi)$, making things considerably simpler. In his 1970 article Ten problems in Hilbert space [19], Halmos asked (Problem 5): "Is every subnormal Toeplitz operator either normal or analytic?"

Among the analytic Toeplitz operators, the most important is the unilateral shift $T(z)$ (here $z\left(e^{i \theta}\right)=e^{i \theta}$ ) which shifts the orthonormal basis of nonnegative frequencies. More generally, a weighted shift is an operator $W$ which acts by shifting some orthonormal basis $\left\{e_{n}: n=0,1,2, \ldots\right\}$ according to the rule $W e_{n}=w_{n} e_{n+1}$; the scalars $w_{n}$ are the weights of $W$. For $T(z)$ and the basis of nonnegative frequencies, one has $w_{n}=1$. In a paper giving a partial yes answer to Halmos's problem [1], Abrahamse asked a prescient question: If a Toeplitz operator is subnormal and is also a weighted shift, must the weight sequence be constant? Sun Shunhua [34] proved that the only possible exceptions (up to constant multiples) form a small family of Toeplitz weighted shifts with the weight sequences $w_{n}=\sqrt{1-\alpha^{2 n+2}}$, where $0<\alpha<1$; the associated orthonormal bases are not the nonnegative frequencies! For each $\alpha$ the corresponding $\phi$, which is continuous and has unit modulus, is $\psi+\alpha \bar{\psi}$, where $\psi$ is a conformal map of the unit disk onto a certain ellipse. Manifestly, $T(\phi)$ is neither normal nor analytic. Cowen and Long provided the final step by showing that these $T(\phi)$ are actually subnormal [12], thus solving Halmos's problem. The problem of describing all subnormal Toeplitz operators remains open.

\section{OTHER DOMAINS, HIGHER DIMENSIONS}

In recent years two new branches of Toeplitz theory have developed, each with its own distinctive flavor. First, one can replace $H^{2}$ by a Bergman space, the space of analytic functions on a complex domain which are square integrable with respect to (possibly weighted) area or volume measure. The Toeplitz operators are again defined as multiplication followed by projection; their properties tend to be determined by the limiting behavior of the symbol near the boundary of the domain. A striking example is the work of Berger and Coburn [4] on the Segal-Bargmann space of entire functions, which provides a Toeplitz model for the creation and annihilation operators of quantum mechanics. Zhu's monograph [40] contains a good introduction.

The second direction, which is not entirely disjoint from the first, might be called topological index theory. Here Toeplitz and related operators act on function spaces on higher-dimensional domains and manifolds; the theory merges into index theory for differential operators. Typically symbols remain continuous and the complication arises in describing Fredholm or other analytical indices via interaction of the symbol with topological or geometric invariants of the domain. A sampling of this active area can be found in Douglas [14], Upmeier [35], and Kaminker [23]. 


\section{The BOOK OF BötTCher AND SilbermanN}

Analysis of Toeplitz operators is a large, friendly book, which will surely be welcomed by all with an interest in the subject. The authors are well known for their contributions to Toeplitz operators, including an earlier monograph on Toeplitz determinants [5]. The emphasis here is on invertibility and Fredholm theory on the circle, although substantial chapters are devoted to Toeplitz operators on the torus $T^{2}$ and to Wiener-Hopf operators on $L^{2}(0, \infty)$. An interesting aspect of the book is the parallel development throughout of Toeplitz theory for $H^{2}$, for the Banach Hardy spaces $H^{p}$, and for the sequence spaces $l^{p}$. Similarly, a systematic treatment of Toeplitz operators with matrix symbols, acting on vector-valued Hardy and sequence spaces, is whenever possible woven through the scalar theory.

Two introductory chapters collect prerequisites and treat the elementary theory of Toeplitz operators on $H^{2}$. The latter category covers a lot of ground, and the presentation is ideal for the analyst who wants to learn the fundamentals. Chapters 3 and 4 form the conceptual core of the book. The authors set forth four broad ideas on which to base their analysis. The first, "algebraization", codifies, in a Banach algebra setting, the main properties of $T$ and related mappings. Algebraization is combined with "essentialization", which means dividing out by some canonical ideal (for example, the compact operators), and "localization", one version of which we have sampled. These algebraic ideas are further mixed with a very general theory of "harmonic approximation", in which one smooths out a discontinuous symbol $\phi$ by integrating against an appropriate approximate identity while retaining sufficient information to extract Fredholm data for $T(\phi)$. Examples are the use of left- and right-hand averages in the treatment of PQC and Fredholm index computation via the winding number, not of $\phi$ (which may not have a winding number), but of Poisson integrals of $\phi$, an idea first applied by Douglas to $H^{\infty}+C$. The generality of the approach makes for some rather elaborate notation which is, nevertheless, well conceived. Also helpful in this regard is the consecutive numbering (within chapters) of all theorems, definitions, remarks, etc. Tracing references to preceding or subsequent material is quite easy.

Two chapters continue themes from the authors' earlier book. The first treats the finite section method for inverting $T(\phi)$, in which one wants to solve the equation $T(\phi) f=g$ by applying the projection $P_{n}$ onto the span $\mathscr{M}_{n}$ of $\left\{1, e^{i \theta}, \ldots, e^{i n \theta}\right\}$. One hopes to solve the matrix equations $P_{n} T(\phi) f_{n}=P_{n} g$ and show that $f_{n} \rightarrow f$ as $n \rightarrow \infty$. The book's final chapter studies determinants of the finite sections $P_{n} T(\phi) \mid \mathscr{M}_{n}$ and their behavior as $n \rightarrow \infty$. This is a timehonored and still-active area with roots in the classical limit theorems of Szegö.

Although the authors have clearly put considerable effort into an abstract framework for their subject, they do not stint on applications to the hard analytical cases. Sarason's theory of PQC, for example, is presented in depth, as is Böttcher's demonstration of the surprising spectral variety attainable with symbols taking on only three distinct values. The reader will also find subtle localization theories of Axler, Clancey, and Gosselin, as well as the authors' proof of a conjecture of Fisher and Hartwig on determinental asymptotics arising from the two-dimensional Ising model. Scattered throughout are many open problems. The authors' fondness for the subject is apparent in this wealth of 
detail. The notes at the end of each chapter, both scholarly and personal in tone, convey the same impression.

Böttcher and Silbermann have brought us a fine book. Though it omits some important topics (most surprisingly, the similarity theory of Clark and Morrel is not mentioned), it is almost encyclopedic within invertibility theory and is a natural sequel to the 1972 text of Douglas [16], which introduced Banach algebra techniques to a wide audience. Moreover, it nicely complements several related books which emphasize different aspects of Toeplitz theory: Rosenblum and Rovnyak [30] (selfadjointness and factorization theory), Nikol'skii [24] (almost everything about the shift operator), and Zhu [40] (Bergman spaces). I expect that Analysis of Toeplitz operators will become a standard reference for both novices and expert practitioners of this beautiful subject.

\section{REFERENCES}

1. M. B. Abrahamse, Subnormal Toeplitz operators and functions of bounded type, Duke J. Math. 43 (1976), 597-604.

2. Anal. 31 (1979), 224-233.

3. S. Axler, S.-Y. A. Chang, and D. Sarason, Products of Toeplitz operators, Integral Equations Operator Theory 1 (1978), 285-309.

4. C. Berger and L. Coburn, Toeplitz operators and quantum mechanics, J. Funct. Anal. 68 (1986), 273-299.

5. A. Böttcher and B. Silbermann, Invertibility and asymptotics of Toeplitz matrices, AkademieVerlag, Berlin, 1983.

6. A. Brown and P. Halmos, Algebraic properties of Toeplitz operators, J. Reine Angew. Math. 213 (1963), 89-102.

7. D. Clark, On Toeplitz operators with loops. II, J. Operator Theory 7 (1982), 109-123.

8. D. Clark and J. Morrel, On Toeplitz operators and similarity, Amer. J. Math. 100 (1978), 973-986.

9. L. Coburn, The $C^{*}$-algebra generated by an isometry. II, Trans. Amer. Math. Soc. 137 (1969), 211-217.

10. L. Coburn and R. Douglas, Translation operators on a half-line, Proc. Nat. Acad. Sci. U.S.A. 62 (1969), 1010-1013.

11. J. Conway, The theory of subnormal operators, Math. Surveys Monographs, vol. 36, Amer. Math. Soc., Providence, RI, 1991.

12. C. Cowen and J. Long, Some subnormal Toeplitz operators, J. Reine Angew. Math. 351 (1984), 216-220.

13. A. Devinatz, Toeplitz operators on $H^{2}$ spaces, Trans. Amer. Math. Soc. 112 (1964), 304317.

14. R. Douglas, Another look at real-valued index theory, Surveys of Some Recent Results in Operator Theory, vol. II (J. Conway and B. Morrel, eds.), Pitman Res. Notes Math. Ser., Longman Sci. Tech., Essex, UK, (co-published with Wiley, New York), pp. 91-120.

15. _ Local Toeplitz operators, Proc. London Math. Soc. (3) 36 (1978), 243-272.

16. __ Banach algebra techniques in operator theory, Academic Press, New York, 1972.

17. I. Gohberg, On Toeplitz matrices constituted by the Fourier coefficients of piecewise continuous functions, Funktsional. Anal. i Prilozhen. 1 (1967), 91-92.

18. I. Gohberg and M. Krein, Systems of integral equations on a half-line with kernels depending on the difference of arguments, Uspekhi Mat. Nauk 13 (1958), 3-72; English transl., Amer. Math. Soc. Transl. Ser. 2, vol. 14, Amer. Math. Soc., Providence, RI, 1960, pp. 217-287. 
19. P. Halmos, Ten problems in Hilbert space, Bull. Amer. Math. Soc. 76 (1970), 887-933.

20. P. Hartman and A. Wintner, On the spectra of Toeplitz matrices, Amer. J. Math. 72 (1950), 359-366.

21. _ The spectra of Toeplitz matrices, Amer. J. Math. 76 (1954), 867-882.

22. R. Ismagilov, The spectrum of Toeplitz matrices, Dokl. Akad. Nauk SSSR 149 (1963), 769772; Soviet Math. Dokl. 4 (1963), 462-465.

23. J. Kaminker, Operator algebraic invariants for elliptic operators, Operator Theory, Operator Algebras and Applications (W. Arveson and R. Douglas, eds.), Proc. Sympos. Pure Math., vol. 51, Part 1, Amer. Math. Soc., Providence, RI, 1990, pp. 307-314.

24. N. K. Nikol'skii, Treatise on the shift operator, "Nauka", Moscow, 1980 (Russian); English transl., Springer-Verlag, Berlin and Heidelberg, 1986.

25. V. Peller, Hankel operators and multivariate stationary processes, Operator Theory, Operator Algebras and Applications (W. Arveson and R. Douglas, eds.), Proc. Sympos. Pure Math., vol. 51, Part 1, Amer. Math. Soc., Providence, RI, 1990, pp. 357-371.

26. S. Power, Essential spectra of piecewise continuous Fourier integral operators, Proc. Royal Irish Acad. Sect. A 18 (1981), 1-7.

27. __ Hankel operators on Hilbert space, Research Notes in Math., vol. 64, Pitman Advanced Publishing Program, Boston, London, and Melbourne, 1982.

28. M. Rosenblum, A concrete spectral theory for self-adjoint Toeplitz operators, Amer. J. Math. 87 (1965), 709-718.

29. __ The absolute continuity of Toeplitz matrices, Pacific J. Math. 10 (1960), 987-996.

30. M. Rosenblum and J. Rovnyak, Hardy classes and operator theory, Oxford Univ. Press, New York and Oxford, 1985.

31. D. Sarason, Toeplitz operators with piecewise quasicontinuous symbols, Indiana Univ. Math. J. 26 (1977), 817-838.

32. B. Silbermann, The $C^{*}$-algebra generated by Toeplitz and Hankel operators with piecewise quasicontinuous symbols, Integral Equations Operator Theory 10 (1987), 730-738.

33. I. Simonenko, The Riemann boundary value problem with measurable coefficients, Dokl. Akad. Nauk SSSR 135 (1960), 538-541; Soviet Math. Dokl. 1 (1960), 1295-1298.

34. Sun Shunhua, Bergman shift is not unitarily equivalent to a Toeplitz operator, Kexue Tongbao 28 (1983), 1027-1030.

35. H. Upmeier, Toeplitz operators and index theory in several complex variables, Operator Theory, Operator Algebras and Applications (W. Arveson and R. Douglas, eds.), Proc. Sympos. Pure Math., vol. 51, Part 1, Amer. Math. Soc., Providence, RI, 1990, pp. 585-598.

36. A. Vol'berg, Two remarks concerning the theorem of S. Axler, S.-Y. A. Chang and D. Sarason, J. Operator Theory 7 (1982), 209-218.

37. H. Widom, Inversion of Toeplitz matrices. II, Illinois J. Math. 4 (1960), 88-99.

38. __ Inversion of Toeplitz matrices. III, Notices Amer. Math. Soc. 7 (1960), 63.

39. _ On the spectrum of Toeplitz operators, Pacific J. Math. 14 (1964), 365-375.

40. K. Zhu, Operator theory on function spaces, Pure Appl. Math. Ser., vol. 139, Marcel Dekker, New York, 1990. 